\title{
Trends and challenges in modeling glioma using 3D human brain organoids
}

\author{
Aruljothi Mariappan ${ }^{1} \cdot$ Gladiola Goranci-Buzhala $^{1} \cdot$ Lucia Ricci-Vitiani $^{2} \cdot$ Roberto Pallini $^{3} \cdot$ Jay Gopalakrishnan $^{1}$
}

Received: 29 September 2020 / Revised: 6 November 2020 / Accepted: 6 November 2020 / Published online: 1 December 2020

(c) The Author(s) 2020. This article is published with open access

\begin{abstract}
The human brain organoids derived from pluripotent cells are a new class of three-dimensional tissue systems that recapitulates several neural epithelial aspects. Brain organoids have already helped efficient modeling of crucial elements of brain development and disorders. Brain organoids' suitability in modeling glioma has started to emerge, offering another usefulness of brain organoids in disease modeling. Although the current state-of-the organoids mostly reflect the immature state of the brain, with their vast cell diversity, human brain-like cytoarchitecture, feasibility in culturing, handling, imaging, and tractability can offer enormous potential in reflecting the glioma invasion, integration, and interaction with different neuronal cell types. Here, we summarize the current trend of employing brain organoids in glioma modeling and discuss the immediate challenges. Solving them might lay a foundation for using brain organoids as a pre-clinical 3D substrate to dissect the glioma invasion mechanisms in detail.
\end{abstract}

\section{Introduction}

Glioma is an aggressive form of brain cancer characterized by poor prognosis [1, 2]. Despite surgical resection, glioma patients succumb due to its rapid growth in the brain, resistance to chemotherapy, and high invasiveness with a median survival time of $\sim 15$ months, and there is no cure. As a result, the 5-year survival rate remains less than 10\% [3-5]. Gliomas possess glioma cancer stem cells (GSCs) that underlie tumor initiation, progression, and recurrence after initial treatment. Furthermore, GSCs exhibit characteristics of neural stem

These authors contributed equally: Aruljothi Mariappan, Gladiola Goranci-Buzhala

Edited by G. Melino

$\triangle$ Jay Gopalakrishnan

jay.gopalakrishnan@hhu.de

1 Institute of Human Genetics, University Hospital Düsseldorf, Heinrich-Heine-Universität, Universitätsstr. 1, 40225 Düsseldorf, Germany

2 Department of Oncology and Molecular Medicine, Istituto Superiore di Sanità, Viale Regina Elena 299, 00161 Rome, Italy

3 Depatment of Neuroscience, Fondazione Policlinico Universitario A. Gemelli, Largo Agostino Gemelli 8, 00168 Rome, Italy cells such as expression of neural stem cell markers and self-renewability. Thus, characterization of GSCs has been critically useful to understand the fundamental biology of glioma [6-10].

GSCs growing in adherent 2D cultures allow studying specific aspects of tumor biology. Furthermore, these 2D GSCs cultures offer possibilities to conduct chemical and genetic screens, transcriptomics, proteomics, clonal or single-cell analyses without the influence of extrinsic signals. Interestingly, early-passaged GSCs retain the genetic and transcriptional signature of the parental tumor, and thus low-passaged GSCs are widely used as an accepted model of glioma [11]. However, studying GSCs in 2D is a particular challenge due to spontaneous changes in stem cell properties and a high degree of stochastic intercellular variations, ultimately resulting in cell populations markedly different from the original tumor. Besides, the invasive behavior of GSCs cannot be modeled in 2D culture, as it requires 3D like brain-like tissue as a host. Therefore, studies have focused on isolating GSCs from primary tumor biopsies and subsequently used them to transplant in mouse models (Patient-derived Xenografts, PDXs). These studies have revealed the surprising plasticity of GSCs reflecting the in vivo tumor heterogeneity [12-14]. Thus, GSCs, when grown in $2 \mathrm{D}$, tend to lose their intrinsic heterogeneity due to the lack of spatial interactions with neighboring cells, extracellular matrix, and tumor microenvironment. As a 
result, drug-screening studies purely relying on $2 \mathrm{D}$ cultures have their downside, as the identified agents may not be therapeutically active in clinical studies.

Last decade researches have seen impressive progress in understanding the fundamental aspects of glioma biology in terms of genetic factors that can elevate intra-tumor heterogeneity, differential behaviors of GSCs, and changes in their growth and invasion behaviors [15-18]. Nevertheless, these efforts have not offered a significant benefit to patients. This could be due to the lack of physiologically relevant a pre-clinical human in vitro system that can reliably recapitulate GSCs invasion behaviors and suitable to adopt robust drug screening studies. Undeniably, a more sophisticated experimental system mirroring the tumor environment, yet allowing experimental manipulations, is required to predict the GSCs behavior accurately. Indeed, recent advances in 3D culturing methods have resulted in so-called "organoids", a 3D structure constituting various cell types allowing their spatio-temporal interactions. On the other hand, when GSCs assemble into glioblastoma organoids (GBOs), they could recapitulate in vivo-like heterogeneity $[19,20]$. While these GBOs can exhibit in vivo-like features, they do not offer the possibility to study the invasion behavior of GSCs in the human brain tissue. While traditional patient-derived xenografts (PDXs) in mice allowed addressing crucial insights into GSCs invasion behavior [21, 22], they lack the human tumor microenvironment. In addition, they fall short because of their lengthy process and incompatible with being applied for drug screening studies [23]. Thus, the lack of suitable in vitro models that mirror the complexities of the human brain is a limiting factor in understanding the fundamental invasion behavior of GSCs in the human brain. In this review, we discuss the applicability of recently emerged 3D human brain organoids in modeling glioma and discussing future perspectives of using them as a pre-clinical model to predict the clinical outcome and use them as a personalized drug-screening platform.

\section{The emergence of human brain organoids and their applications}

Brain organoids are an innovative experimental model of modern reductionists' approach. It is noteworthy that the early developmental neurobiologists in the last century have already adapted similar deconstructive strategies to deconstruct the complexities of human brain development $[24,25]$. In the past, researchers did not have access to currently available pure cultures of pluripotent cells, but their approaches relied on ex vivo tissues that already harbored resident stem cells. Thus, with the combination of now available embryonic stem cells (ES) or induced pluripotent cells (iPSCs), modern 3D culturing technologies and directed differentiation protocols have enabled us to exploit the self-organization ability of pluripotent stem cells to generate human brain-like tissues named brain organoids. These 3D tissues recapitulate many aspects of neural epithelial cells cytoarchitecturally similar to the developing human brain. The historical aspects of different experiments that led to the generation of modern-day brain organoids are described elsewhere [26, 27].

In this modern era of brain organoids, two laboratories stand out to receive the full credit for their pioneering effort in generating the first kind of 3D neural tissues in vitro. Attempts by the Sasai and Vaccarino laboratories have generated self-organized structures from pluripotent cells that recapitulated several neuroretina and telencephalic development [28-30]. These neural tissues stunningly displayed a brain-like architecture constituting polarized radial glia, intermediate progenitors, and layer-specific cortical neurons similar to their in vivo tissues' cytoarchitecture. Their seminal works have defined self-assembly principles and laid the foundations for the number of laboratories to generate so-called "3D human brain organoids" [31, 32]. Technological developments by other labs have then brought brain organoids into the limelight, which helped others decoding the early events of brain development, including interneuron migration and cell diversity analysis [33-35]. In this context, the Gopalakrishnan lab has utilized early brain organoids to dissect microcephaly mechanisms due to genetic mutation and identified shared mechanisms of microcephaly due to genetic mutation and Zika virus infections [36-38]. The Guo Li-Ming lab has devised a method to generate region-specific brain organoids that have further refined target cell types of Zika virus [39]. The Arlotta lab revealed the extensive cell diversities of human brain organoids [35]. Lately, brain organoids have also surprisingly helped to model neurological COVID-19 [40]. Several elegant works have further shined the original brain organoid generation protocols, which we apologize for not mentioning here due to lack of space. Many review works have comprehensively listed various kinds of brain organoids, methods, and their use ranging from classical developmental biology to modeling pathomechanisms of multiple diseases, including glioma [27, 41-44]

\section{Do human brain organoids suitable 3D host tissues for GSCs?}

Unlike other cancer types, gliomas metastasize rarely but infiltrates diffusively into the brain $[45,46]$. Thus, despite the region-specific resection of glioma tumors, the diffused glioma spreading into the rest of the brain tissue is persistent. Clinically, this is challenging and is a significant 
bottleneck in therapeutics. 2D cultures do not help to assay such an invasive behavior of GSCs. Therefore; it became essential to model the diffusiveness of GSCs in brain mimetics using $3 \mathrm{D}$ brain organoids as it may provide the first glimpse of GSCs' behavior in the host tissues. Imaging at high resolution, the infiltration assay may map the migratory characteristics of GSCs in the human brain. Finally, one can optimize and upscale the assay that can be extended as personalized glioma invasion and drug screening assays. As described before, only a handful of studies have just started to recognize brain organoids' power to study the glioma invasion mechanisms.

Bian and colleagues explored the use of genetically engineered brain organoids and expressed oncogenes ectopically to develop tumors. The observations of ectopic growth of tumor-like mass in organoids did not sufficiently help assessing to what extent those cells' invasion behaviors mirror the biological relevance [47]. More recently, Ballabio et al. used human cerebellar organoids to understand the molecular mechanisms of medulloblastoma development and identified that Otx2 and c-MYC as inducers of medulloblastoma [48]. Interestingly, the authors have extended their system to assay the anti-tumor activity of Tazemetostat, an EZH2-specific inhibitor that could antagonize Otx2/c-MYC-mediated tumorigenesis. Their study identifies the usefulness of brain organoids in identifying deregulated pathways of tumor formation. Ogawa et al., on the other hand, made a further refinement in the assay by implanting patient-derived glioma cells and provided an insight that the foreign materials can establish tumor-like structures in the human brain organoids [49]. The Fine lab then used human embryonic stem cell (hESC)-derived brain organoids to host clinical glioma samples and showed that the behaviors of GSCs such as tissue invasion and tumor formations, phenocopying patient gliomas. Their work also provided the first glimpses of GSCspenetrating organoids for chemo and radiotherapy, thereby setting how one can exploit the brain organoids for glioma modeling [50]. In summary, both of these initial works have highlighted an important point that GSCs have preferential tropism to organoid tissues even though brain organoids are far from the human brain tissue in terms of mature cell types and vasculatures.

To further optimize the GSCs invasion assay, GoranciBuzhala et al. tested various parameters such as organoid's age, GSC numbers, and the order of GSCs addition either single cells or spheres [51]. Their attempts have devised a rapid and efficient glioma modeling. Importantly, their assays can distinguish different invasion behaviors of primary and recurrent patient derived GSCs. Tissue clearing and high-resolution quantitative 3D imaging have revealed unbiased identification and quantifications of invasion protrusions, depth of invasion, and volume occupied within the host tissues of brain organoids. Strikingly, these assays have shown a surprising finding that GSCs invasion's propensity was inversely co-related to organoids age, indicating that mature neuronal types can provide a favoring microenvironment for the GSCs. Earlier studies have shown that neuronal activities release mitogenic factors that can support glioma cell proliferation in the host brain tissues [52]. Trying to mimic this aspect, Goranci-Buzhala et al. took an orthogonal approach by providing synaptic protein Neuroligin-3 (NLGN-3) exogenously. They identified that indeed NLGN-3 could enhance the invasion behavior of GSCs in early-stage organoids, which are predominantly at the stage of active proliferation [51]. All of these findings provide surprising hints that brain organoids can provide an ex-vivo-like experimental system enabling to map the invasion behavior of GSCs quantitatively.

Based on the observations, it is intuitive that GSCs not only invade into the brain but also interact with the neuronal cells to integrate and to exert its effect to disrupt normal brain functions. To test this aspect, Goranci-Buzhala, and colleagues cultured organoid slices that could generate long-range axons growing as neuronal outgrowths [51]. Importantly, these neurons represented mature neuronal cell types [36]. GSCs surprisingly interacted with neurons establishing hemisynapses-like structures [51]. Strikingly, similar GSCsneuronal interactions have also been recently described in mouse xenografts [53]. It remains to be tested whether the integrated GSCs human brain organoids exhibit electric activities. Figure 1 schematically summarizes the current state of glioma invasion assays in human brain organoids.

In summary, even though brain organoid-based glioma invasion assays still at the level of development, the findings so far are encouraging that brain organoid tissues can serve as a 3D substrate for clinical GSCs. Nevertheless, the field requires significant refinements, which we outline in the following section. Table 1 summarizes the currently available 3D culture systems to study glioma invasion biology.

\section{Challenges in organoid-based glioma modeling}

\section{Lack of mature neuronal and non-neuronal cell types}

As described previously, stem cell-derived brain organoids is a reductionist approach containing mostly immature cell types of developing brain. Thus, these organoids perfectly fit to decode the rules that determine neural stem cell proliferation, differentiation, and primitive cortical plate formation in brain development. Glioma is a late-onset disorder, and thus more meaningful glioma modeling requires brain organoids harboring mature cell types of astrocytes, oligodendrocytes, myelinated neurons, and 

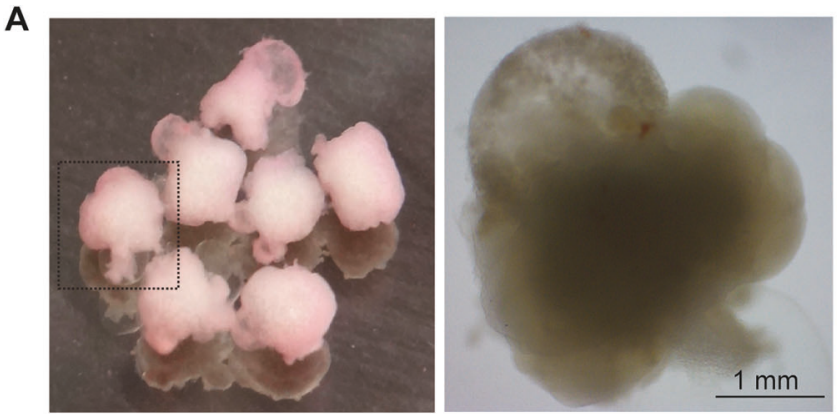

(Gabriel et al., 2016, The EMBO journal 35(8):803-19)

B

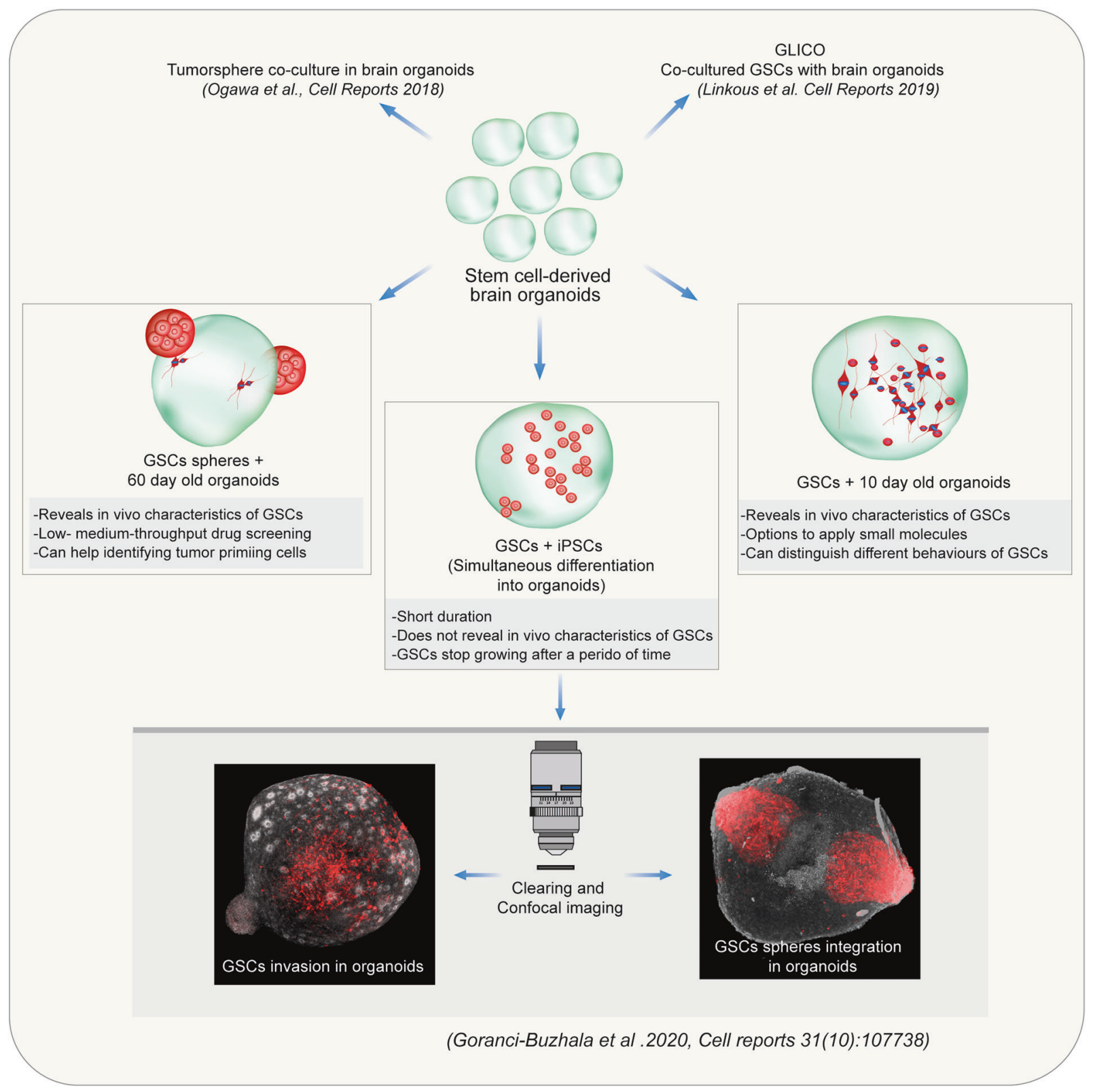

Fig. 1 Examples of iPSCs-derived human brain organoids. A Brain organoids shown here exhibits morphologically similar appearances of human neural epithelial tissues with fluid-filled regions. The images adopted from Gabriel et al. EMBO Journal 2016. B Schematic illustrations of various glioma invasion assays that used patient derived GSCs and human brain organoids. Schemes also explain different methods of optimization and what they can reveal. 


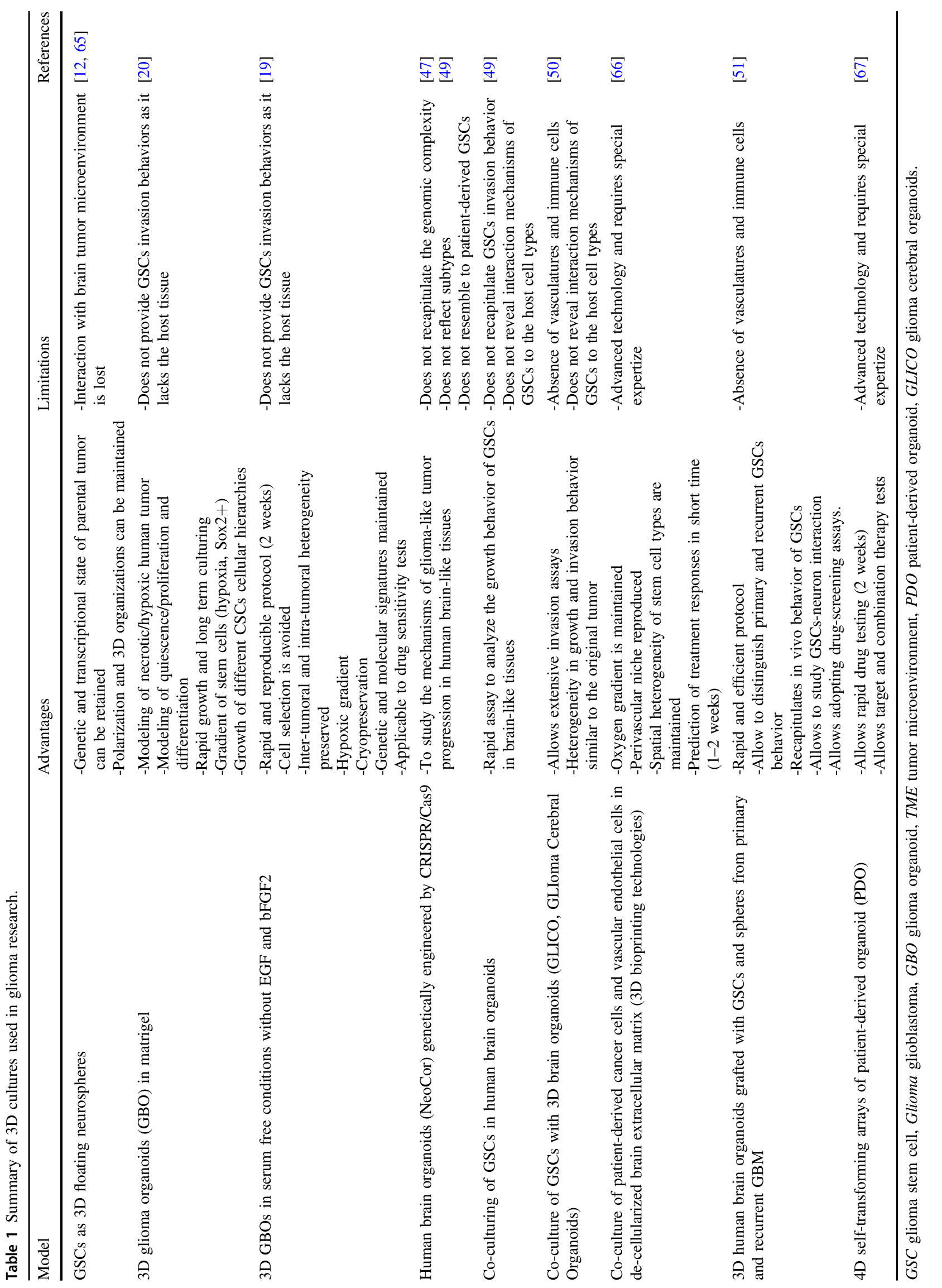


immune defense cell types of microglia. It is particularly important to employ organoids with a myriad of cell types because GSCs require a microenvironment. GSCs communicate, reprogram the surrounding cells, suppress the immune response, and remodel the extracellular matrix, events that all favor tumor progression. 3D tissues merely containing actively proliferating precursor cells may help obtain initial events but will not provide complex interactions occurring in glioma modeling.

To date, several protocols that generate brain organoids allow the differentiation of astrocytes, mature neuronal cell types, and even surprisingly microglia-like cells [54, 55]. Microglia are essential cell types as glioma cells communicate with them via releasing extracellular vesicles [56, 57]. Microglial cells in brain organoids are unexpected, as microglial cells do not originate from neuroectoderm, which is the primary germline to generate neural lineages. Dual-SMAD inhibition is a mechanism that can trigger the neuroectoderm formation. Ormel et al. took a thoughtful approach of generating brain organoids omitting SMAD inhibitors, which surprisingly developed Iba-1-positive microglial cells with their characteristics of ramified morphology [58]. Omitting retinoic acid at the initial stage of differentiation condition, Ramani et al. have also observed microglial cells and astrocytes in their organoids [40]. Even though it is difficult to explain the origin of non-ectodermal related cell types in directed differentiation conditions, brain organoids appear to have some degree of plasticity depending on the differentiation cues.

\section{Lack of endothelial cells and vasculatures}

Another critical missing factor in the brain organoids is endothelial cells. It is known that GSCs reside preferentially in the vascular niches where they establish a spatial connection with endothelial cells that could affect their sensitivity to chemotherapy [59, 60]. When cocultured with endothelial cells, brain organoids generate hybrid organoids of neuroepithelial tissue vasculatures. However, the In-Hyun Park lab devised an elegant method to generate functional vasculatures in an inducible manner by expressing ETS variant 2 that reprograms dermal fibroblasts into endothelial cells [61]. Even more surprisingly, the generated vasculature-like structures improved the organoid qualities in terms of maturation, exhibiting blood-brain barrier characteristics.

\section{Lack of a method that can generate organoids in large quantities}

Another critical development in organoid technology is to devise a method that can generate a large number of brain organoids with minimum inter-organoid variations. In our opinion, this effort is particularly critical when organoids are used for drug screening and personalized glioma invasion assays, both of which require statistically significant data. In this direction, using a large scRNA-seq dataset, Velasco et al. demonstrated a reliable reproducibility between individual organoids that they generated [62]. Amending their approach with a method that can generate large quantities of organoids will yield a scaling-up process that can generate a large number of organoids exhibiting minimum inter-organoid variations within a batch. One of the limiting factors that cause inter-organoid variations is that the organoid generation method involves numerous steps manually, such as dispensing cells, embedding them in the matrix, and generating organoid intermediates such as embryoid bodies and neurospheres. These steps could cause aberrations, which may impact the overall homogeneity of organoids. Perhaps future semi-automated dispensing, employing pre-patterned microsphere wells and directed differentiation conditions skipping intermediate steps can help generating large numbers of brain organoids harboring disease-relevant cell types for efficient glioma modeling.

\section{Glioma tumoroids or GSCs?}

The outcome of the glioma invasion assay depends on the starting material. As discussed above, low-passaged GSCs are accepted models of glioma [14]. However, extended passaging and altered culturing conditions can impact their heterogeneity and behavior due to selective clonal evolution in 2D cultures. An alternative to single cell GSCs is glioma tumoroids, which can be generated directly from the clinical samples by culturing them under conditions that maintain the stem cell niches. These glioma tumoroids do not differ from currently described GBOs (Glioblastoma organoids) [19]. We chose to use the term "tumoroids" since the resulting 3D objects directly emerge from clinical tumors not from self-assembling pluripotent cells. On the other hand, organoids per se mean a self-assembled 3D objects via controlled differentiation of pluripotent cells. As recently described, these glioma tumoroids mirror the histological features, complex cellular diversities, genetic signatures, and mutational profiles of their original tumor. Moreover, these glioma tumoroids can be generated quite rapidly and do not require repeated culturing in 2D. Most strikingly, when implanted in rodent brains, these glioma tumoroids exhibit aggressive infiltration in to surrounding tissues. Therefore, these 3D glioma tumoroids may be superior to GSCs in preserving the heterogeneity of parent tumor tissue. The described glioma tumoroids may contain infiltrating cells and constitute cell types that can support the infiltrating cells. Future experiments can test this aspect by conducting side-by-side comparative invasion 
experiments with glioma tumoroids and GSCs isolated from these tumoroids.

\section{Future perspectives; from simple glioma invasion assay to personalized medicine}

Brain organoid models are beneficial for bridging the knowledge gap between in vitro cell cultures and in vivo animal studies. Even though GSCs are accepted models of glioma biology, GSCs may not faithfully reflect the characteristics of parent tumors. There are numerous reasons for it: (i) the culturing conditions can potentially out select a population, (ii) the artifacts of culturing conditions may suppress the heterogeneity of initial tumors, (iii) multiple passages may change the behaviors of GSCs. The glioma tumoroids can significantly address these pitfalls. When brain organoid-based glioma invasion assay is optimized with glioma tumoroids, it will be possible to scale up the reproducible assay with a reasonable turnaround time between four to six weeks after the surgery.

Besides, high-resolution imaging and computer algorithmassisted post-processing should be in place to generate a reliable invasion map of a given clinical sample. Establishing these measures will ensure implementing the assay in a clinical setting. It is noteworthy that brain organoid invasion assays already allow distinguishing the behavior of primary and recurrent GSCs [51]. Thus, an optimized assay would enable us to stratify glioma patients based on the genetic background and the invasion behavior of patient specific GSCs. All of these developments will lay foundations to implement personalized brain organoid-based glioma invasion assays.

Brain organoid-based invasion assays hold promise for personalized drug discovery programs. Traditional treatment for glioma is the surgical resection of naïve tumors followed by radiation in combination with temozolomide (TMZ). It may be possible that organoid-based invasion assays allow us to predict the drug sensitivity of the naïve GSCs before exposure to radiation and TMZ. It is important note that drugs targeting cell cycle pathways might also affect the proliferative cells of immature organoids. To avoid this situation, one could use mature old organoids or organoid-like structures assembled from post mitotic neurons and astrocytes [63]. In the clinical setting, physicians often face glioma recurring after initial therapy. It is noteworthy that most gliomas accumulate genetic changes already at their therapy naïve status that is before the firstline treatment. Only a minor genetic change occurs in the recurrent tumor after the first-line treatment [64]. In spite of this, radio-chemotherapy is likely to affect genetic backgrounds, drug sensitivity of GSCs, and invasive behavior. For example, Goranci-Buzhala et al. described that their organoid-based glioma invasion assays had provided the first hints that recurrent GSCs are much more infiltrative in brain organoids than the GSCs derived from primary surgery [51]. It will be attractive if the described assays are scaled up to set up medium-throughput to high-throughput assays to identify agents that can mitigate the GSCs invasion. For example, identifying a battery of repurposing drugs that can impair patient derived GSCs invasion in brain organoids will greatly help therapeutics options at the clinic.

There is also a room for developing tools to conduct autologous glioma invasion assays. Currently used brain organoid-based invasion assays are performed in a homologous manner, meaning that patient-derived GSCs are grafted in different host brain organoids. Thus, another significant development is establishing autologous innovation assays by grafting patient derived GSCs into brain organoids generated from the same patient. Comparative invasion assays between homologous and autologous assays may reveal the role of diverse human brain microenvironments on tumor spread.

\section{Conclusion and outlook}

The recently emerged 3D human brain organoids have unexpectedly offered us studying glioma biology in a new dimension, offering an excellent opportunity to visualize GSCs invasion in human brain-like tissues. With its neural stem cells, early and mature neurons, astrocytes, and microglial cells, brain organoids provide a suitable brainlike microenvironment for GSCs growth. Human brain organoids did not exist in the last decade, and organoidbased GSCs invasion assays were not there just three years ago. Hence, it is an exciting era for glioma researchers as one can map GSCs invasion in ex-vivo. However, as we discussed in the previous chapter, there are significant bottlenecks, and addressing them will allow us to model the vicious cycle of GSCs, such as the aggressive behavior of GSCs along white matter paths of the human brain (like corona radiates), peri-vascular invasion/niching and escape from immunological surveillance. From a clinical standpoint, optimizing the assays is crucial to identify responses on the sensitivity of a given tumor sample to irradiation or drug treatments within a few weeks of surgery. Such early responses will help predict patient survival after standard treatment and, hopefully, tailor personalized therapies. These innovations await future experiments.

Acknowledgements This work was financially supported by grants from the research commision of Heinrich-Heine-Universität, Düsseldorf to J.G and from AIRC (IG 2019 number 23154) to R.P.

Funding Open Access funding enabled and organized by Projekt DEAL. 


\section{Compliance with ethical standards}

Conflict of interest The authors declare that they have no conflict of interest.

Publisher's note Springer Nature remains neutral with regard to jurisdictional claims in published maps and institutional affiliations.

Open Access This article is licensed under a Creative Commons Attribution 4.0 International License, which permits use, sharing, adaptation, distribution and reproduction in any medium or format, as long as you give appropriate credit to the original author(s) and the source, provide a link to the Creative Commons license, and indicate if changes were made. The images or other third party material in this article are included in the article's Creative Commons license, unless indicated otherwise in a credit line to the material. If material is not included in the article's Creative Commons license and your intended use is not permitted by statutory regulation or exceeds the permitted use, you will need to obtain permission directly from the copyright holder. To view a copy of this license, visit http://creativecommons. org/licenses/by/4.0/.

\section{References}

1. Stupp R, Mason WP, van den Bent MJ, Weller M, Fisher B, Taphoorn MJ, et al. Radiotherapy plus concomitant and adjuvant temozolomide for glioblastoma. N. Engl J Med. 2005;352:987-96.

2. Burnet NG, Lynch AG, Jefferies SJ, Price SJ, Jones PH, Antoun NM, et al. High grade glioma: imaging combined with pathological grade defines management and predicts prognosis. Radiother Oncol. 2007;85:371-8.

3. Furnari FB, Fenton T, Bachoo RM, Mukasa A, Stommel JM, Stegh A, et al. Malignant astrocytic glioma: genetics, biology, and paths to treatment. Genes Dev. 2007;21:2683-710.

4. Buckner JC. Factors influencing survival in high-grade gliomas. Semin Oncol. 2003;30:10-4.

5. Rusthoven CG, Koshy M, Sher DJ. Radiation plus temozolomide in patients with glioblastoma. N. Engl J Med. 2017;376:2195-7.

6. Swartling FJ, Cancer M, Frantz A, Weishaupt H, Persson AI. Deregulated proliferation and differentiation in brain tumors. Cell Tissue Res. 2015;359:225-54.

7. Alcantara Llaguno SR, Xie X, Parada LF. Cell of origin and cancer stem cells in tumor suppressor mouse models of glioblastoma. Cold Spring Harb Symposia Quant Biol. 2016;81:31-6.

8. D'Alessandris QG, Biffoni M, Martini M, Runci D, Buccarelli M, Cenci $\mathrm{T}$, et al. The clinical value of patient-derived glioblastoma tumorspheres in predicting treatment response. Neuro-Oncol. 2017;19:1097-108.

9. Azzarelli R, Simons BD, Philpott A. The developmental origin of brain tumours: a cellular and molecular framework. Development. 2018;145:dev162693.

10. Hakes AE, Brand AH. Neural stem cell dynamics: the development of brain tumours. Curr Opin Cell Biol. 2019;60:131-8.

11. Zanders ED, Svensson F, Bailey DS. Therapy for glioblastoma: is it working? Drug Discov Today. 2019;24:1193-201.

12. Galli R, Binda E, Orfanelli U, Cipelletti B, Gritti A, De Vitis S, et al. Isolation and characterization of tumorigenic, stem-like neural precursors from human glioblastoma. Cancer Res. 2004;64: 7011-21.

13. Singh SK, Hawkins C, Clarke ID, Squire JA, Bayani J, Hide T, et al. Identification of human brain tumour initiating cells. Nature. 2004;432:396-401.
14. Pollard SM, Yoshikawa K, Clarke ID, Danovi D, Stricker S, Russell R, et al. Glioma stem cell lines expanded in adherent culture have tumor-specific phenotypes and are suitable for chemical and genetic screens. Cell Stem Cell. 2009;4:568-80.

15. Bergmann N, Delbridge C, Gempt J, Feuchtinger A, Walch A, Schirmer L, et al. The intratumoral heterogeneity reflects the intertumoral subtypes of glioblastoma multiforme: a regional immunohistochemistry analysis. Front Oncol. 2020;10:494.

16. Neftel C, Laffy J, Filbin MG, Hara T, Shore ME, Rahme GJ, et al. An integrative model of cellular states, plasticity, and genetics for glioblastoma. Cell. 2019;178:835-49 e21.

17. Sottoriva A, Spiteri I, Piccirillo SG, Touloumis A, Collins VP, Marioni JC, et al. Intratumor heterogeneity in human glioblastoma reflects cancer evolutionary dynamics. Proc Natl Acad Sci USA. 2013;110:4009-14.

18. Gerlinger M, Rowan AJ, Horswell S, Math M, Larkin J, Endesfelder $\mathrm{D}$, et al. Intratumor heterogeneity and branched evolution revealed by multiregion sequencing. N. Engl J Med. 2012;366:883-92.

19. Jacob F, Salinas RD, Zhang DY, Nguyen PTT, Schnoll JG, Wong $\mathrm{SZH}$, et al. A patient-derived glioblastoma organoid model and biobank recapitulates inter- and intra-tumoral heterogeneity. Cell. 2020;180:188-204 e22.

20. Hubert CG, Rivera M, Spangler LC, Wu Q, Mack SC, Prager BC, et al. A three-dimensional organoid culture system derived from human glioblastomas recapitulates the hypoxic gradients and cancer stem cell heterogeneity of tumors found in vivo. Cancer Res. 2016;76:2465-77.

21. Xie Y, Bergstrom T, Jiang Y, Johansson P, Marinescu VD, Lindberg N, et al. The human glioblastoma cell culture resource: validated cell models representing all molecular subtypes. EBioMedicine. 2015;2:1351-63.

22. Joo KM, Kim J, Jin J, Kim M, Seol HJ, Muradov J, et al. Patientspecific orthotopic glioblastoma xenograft models recapitulate the histopathology and biology of human glioblastomas in situ. Cell Rep. 2013;3:260-73.

23. Aldape K, Brindle KM, Chesler L, Chopra R, Gajjar A, Gilbert $\mathrm{MR}$, et al. Challenges to curing primary brain tumours. Nat Rev Clin Oncol. 2019;16:509-20.

24. Harrison RG. Observations on the living developing nerve fiber. Proc Soc Exp Biol Med,. 1906;4:140-3. https://doi.org/10.3181/ 00379727-4-98.

25. Holtfreter J. Neural differentiation of ectoderm through exposure to saline solution. J Exp Zool. 1944;95:307-44.

26. Kim J, Koo BK, Knoblich JA. Human organoids: model systems for human biology and medicine. Nat Rev Mol cell Biol. 2020;21:571-84.

27. Schutgens F, Clevers H. Human organoids: tools for understanding biology and treating diseases. Annu Rev Pathol. 2020;15: 211-34.

28. Eiraku M, Watanabe K, Matsuo-Takasaki M, Kawada M, Yonemura S, Matsumura M, et al. Self-organized formation of polarized cortical tissues from ESCs and its active manipulation by extrinsic signals. Cell Stem Cell. 2008;3:519-32.

29. Mariani J, Simonini MV, Palejev D, Tomasini L, Coppola G, Szekely AM, et al. Modeling human cortical development in vitro using induced pluripotent stem cells. Proc Natl Acad Sci USA. 2012;109:12770-5.

30. Kadoshima T, Sakaguchi H, Nakano T, Soen M, Ando S, Eiraku $\mathrm{M}$, et al. Self-organization of axial polarity, inside-out layer pattern, and species-specific progenitor dynamics in human ES cell-derived neocortex. Proc Natl Acad Sci USA. 2013;110: 20284-9.

31. Renner M, Lancaster MA, Bian S, Choi H, Ku T, Peer A, et al. Self-organized developmental patterning and differentiation in cerebral organoids. EMBO J. 2017;36:1316-29. 
32. Birey F, Andersen J, Makinson CD, Islam S, Wei W, Huber N, et al. Assembly of functionally integrated human forebrain spheroids. Nature. 2017;545:54-9.

33. Jo J, Xiao Y, Sun AX, Cukuroglu E, Tran HD, Goke J, et al. Midbrain-like organoids from human pluripotent stem cells contain functional dopaminergic and neuromelanin-producing neurons. Cell Stem Cell. 2016;19:248-57.

34. Xiang Y, Tanaka Y, Patterson B, Kang YJ, Govindaiah G, Roselaar N, et al. Fusion of regionally specified hPSC-derived organoids models human brain development and interneuron migration. Cell Stem Cell. 2017;21:383-98 e7.

35. Quadrato G, Nguyen T, Macosko EZ, Sherwood JL, Min Yang S, Berger DR, et al. Cell diversity and network dynamics in photosensitive human brain organoids. Nature. 2017;545:48-53.

36. Gabriel E, Wason A, Ramani A, Gooi LM, Keller P, Pozniakovsky A, et al. CPAP promotes timely cilium disassembly to maintain neural progenitor pool. EMBO J. 2016;35:803-19.

37. Gabriel E, Ramani A, Karow U, Gottardo M, Natarajan K, Gooi $\mathrm{LM}$, et al. Recent Zika virus isolates induce premature differentiation of neural progenitors in human brain organoids. Cell Stem Cell. 2017;20:397-406.

38. Gabriel E, Gopalakrishnan J. Generation of iPSC-derived Human Brain Organoids to Model Early Neurodevelopmental Disorders. Journal of visualized experiments. JoVE. 2017;122:e55372.

39. Qian X, Nguyen HN, Song MM, Hadiono C, Ogden SC, Hammack C, et al. Brain-region-specific organoids using minibioreactors for modeling ZIKV exposure. Cell. 2016;165: 1238-54.

40. Ramani A, Muller L, P NO, Gabriel E, Abida-Islam P, MullerSchiffmann A, et al. SARS-CoV-2 targets neurons of 3D human brain organoids. EMBO J. 2020;39:e106230.

41. Azzarelli R. Organoid models of glioblastoma to study brain tumor stem cells. Front Cell Dev Biol. 2020;8:220.

42. Marton RM, Pasca SP. Organoid and assembloid technologies for investigating cellular crosstalk in human brain development and disease. Trends Cell Biol. 2020;30:133-43.

43. Gabriel E, Ramani A, Altinisik N, Gopalakrishnan J. Human brain organoids to decode mechanisms of microcephaly. Front Cell Neurosci. 2020;14:115.

44. Gopalakrishnan J. The emergence of stem cell-based brain organoids: trends and challenges. BioEssays 2019;41:e1900011.

45. Barthel FP, Johnson KC, Varn FS, Moskalik AD, Tanner G, Kocakavuk E, et al. Longitudinal molecular trajectories of diffuse glioma in adults. Nature. 2019;576:112-20.

46. Ceccarelli M, Barthel FP, Malta TM, Sabedot TS, Salama SR, Murray BA, et al. Molecular profiling reveals biologically discrete subsets and pathways of progression in diffuse glioma. Cell. 2016;164:550-63.

47. Bian S, Repic M, Guo Z, Kavirayani A, Burkard T, Bagley JA, et al. Genetically engineered cerebral organoids model brain tumor formation. Nat Methods. 2018;15:631-9.

48. Ballabio C, Anderle M, Gianesello M, Lago C, Miele E, Cardano $\mathrm{M}$, et al. Modeling medulloblastoma in vivo and with human cerebellar organoids. Nat Commun. 2020;11:583.

49. Ogawa J, Pao GM, Shokhirev MN, Verma IM. Glioblastoma model using human cerebral organoids. Cell Rep. 2018;23:1220-9.
50. Linkous A, Balamatsias D, Snuderl M, Edwards L, Miyaguchi K, Milner T, et al. Modeling patient-derived glioblastoma with cerebral organoids. Cell Rep. 2019;26:3203-11 e5.

51. Goranci-Buzhala G, Mariappan A, Gabriel E, Ramani A, RicciVitiani L, Buccarelli M, et al. Rapid and efficient invasion assay of glioblastoma in human brain organoids. Cell Rep. 2020;31:107738.

52. Venkatesh HS, Johung TB, Caretti V, Noll A, Tang Y, Nagaraja $\mathrm{S}$, et al. Neuronal activity promotes glioma growth through neuroligin-3 secretion. Cell. 2015;161:803-16.

53. Venkatesh HS, Morishita W, Geraghty AC, Silverbush D, Gillespie SM, Arzt M, et al. Electrical and synaptic integration of glioma into neural circuits. Nature. 2019;573:539-45.

54. Sloan SA, Andersen J, Pasca AM, Birey F, Pasca SP. Generation and assembly of human brain region-specific three-dimensional cultures. Nat Protoc. 2018;13:2062-85.

55. Pasca AM, Sloan SA, Clarke LE, Tian Y, Makinson CD, Huber $\mathrm{N}$, et al. Functional cortical neurons and astrocytes from human pluripotent stem cells in 3D culture. Nat Methods. 2015;12:671-8.

56. Abels ER, Maas SLN, Nieland L, Wei Z, Cheah PS, Tai E, et al. Glioblastoma-associated microglia reprogramming is mediated by functional transfer of extracellular miR-21. Cell Rep. 2019;28: 3105-19 e7.

57. Hambardzumyan D, Gutmann DH, Kettenmann H. The role of microglia and macrophages in glioma maintenance and progression. Nat Neurosci. 2016;19:20-7.

58. Ormel PR, Vieira de Sa R, van Bodegraven EJ, Karst H, Harschnitz O, Sneeboer MAM, et al. Microglia innately develop within cerebral organoids. Nat Commun. 2018;9:4167.

59. Onishi M, Ichikawa T, Kurozumi K, Date I. Angiogenesis and invasion in glioma. Brain Tumor Pathol. 2011;28:13-24.

60. Tate MC, Aghi MK. Biology of angiogenesis and invasion in glioma. Neurotherapeutics. 2009;6:447-57.

61. Cakir B, Xiang Y, Tanaka Y, Kural MH, Parent M, Kang YJ, et al. Engineering of human brain organoids with a functional vascular-like system. Nat Methods. 2019;16:1169-75.

62. Velasco S, Kedaigle AJ, Simmons SK, Nash A, Rocha M, Quadrato $\mathrm{G}$, et al. Individual brain organoids reproducibly form cell diversity of the human cerebral cortex. Nature. 2019;570:523-7.

63. Liu SJ, Malatesta M, Lien BV, Saha P, Thombare SS, Hong SJ, et al. CRISPRi-based radiation modifier screen identifies long non-coding RNA therapeutic targets in glioma. Genome Biol. 2020;21:83.

64. Korber V, Yang J, Barah P, Wu Y, Stichel D, Gu Z, et al. Evolutionary trajectories of IDH(WT) glioblastomas reveal a common path of early tumorigenesis instigated years ahead of initial diagnosis. Cancer Cell. 2019;35:692-704 e12.

65. Azari H, Millette S, Ansari S, Rahman M, Deleyrolle LP, Reynolds BA. Isolation and expansion of human glioblastoma multiforme tumor cells using the neurosphere assay. J Vis Exp. 2011;56:e3633.

66. Yi HG, Jeong YH, Kim Y, Choi YJ, Moon HE, Park SH, et al. A bioprinted human-glioblastoma-on-a-chip for the identification of patient-specific responses to chemoradiotherapy. Nat Biomed Eng. 2019;3:509-19.

67. Chadwick M, Yang C, Liu L, Gamboa CM, Jara K, Lee H, et al. Rapid processing and drug evaluation in glioblastoma patientderived organoid models with 4D bioprinted arrays. iScience. 2020;23:101365. 